\title{
Pesquisa em educação: Um panorama bibliométrico de publicações em Língua Portuguesa, no Scielo, no período de 2002 a 2020
}

\author{
Isabel Pinho1', Eduardo Amadeu Dutra Moresi² e Mário de Oliveira \\ Braga Filho ${ }^{2}$
}

\author{
1 Universidade de Aveiro, Portugal | isabelpinho@ua.pt | http://orcid.org/0000-0003-1714- \\ 8979 \\ 2 Programa de Mestrado e Doutorado em Educação da Universidade Católica de Brasília \\ Brasil | moresi@p.ucb.br; braga@p.ucb.br; | https://orcid.org/0000-0001-6058-3883; \\ https://orcid.org/0000-0003-3563-0590
}

\begin{abstract}
Resumo: A pesquisa em educação é um campo muito amplo, devido às diversas instâncias da gestão educacional e aos inúmeros sujeitos da educação, aos distintos processos e metodologias educacionais e aos projetos e políticas de educação, inseridos em uma conjuntura de mudanças sociais, econômicas e políticas que se operam na atualidade, nos planos nacional e internacional. Este trabalho apresenta panorama das publicações sobre pesquisa em educação por meio de uma análise bibliométrica, para responder à questão de pesquisa, explorando a análise de redes de coocorrência de palavras-chave e de citações de documentos, de periódicos e de organizações. A pesquisa bibliográfica foi realizada na base Scielo Citation Index (Web of Science) utilizando a expressão de busca: educação, que retornou 23731 documentos publicados do período de 2002 a 2021. Foram realizados três refinamentos da pesquisa com a seleção: da categoria - education educational research; dos tipos de documentos - artigos em periódicos e de revisão; e do idioma - português. O resultado obteve 7448 documentos publicados no período de 2002 a 2021. O panorama foi obtido da análise das redes de coocorrência de palavras-chave, de citações de documentos, de citações de fontes e de organizações.
\end{abstract}

Palavras-chave: Pesquisa em Educação; Bibliometria; Análise de Coocorrência; Análise de Citações.

\section{Educational Research: A Bibliometric Overview of the Documents Published in Portuguese in Scielo from 2002 to 2020}

\begin{abstract}
Educational research is a very broad field, due to the various instances of educational management and the numerous subjects of education, the different educational processes and methodologies and the projects and policies of education, inserted in a conjuncture of social, economic and political changes that currently operate, nationally and internationally. This paper presents an overview of publications on educational research through a bibliometric analysis, to answer the research question, exploring the analysis of cokeywords co-occurrence networks and documents, sources and organizations citation networks. The bibliographic search was carried out using the Scielo Citation Index (Web of Science) using the search term: education, which returned 23731 documents published from 2002 to 2021. Three refinements of the research were carried out with the selection: from the category - education educational research; types of documents - articles in journals and reviews; and the language - portuguese. The result obtained 7448 documents published in the period from 2002 to 2021. The overview was obtained from the analysis of the keyword cooccurrence networks and the documents, sources and organizations citation networks.
\end{abstract}

Keywords: Educational Research; Bibliometrics; Co-occurrence Analysis; Citation Analysis.

\section{Introdução}

A pesquisa em educação (ou investigação em educação) é um campo muito amplo, devido às diversas instâncias da gestão educacional, aos inúmeros sujeitos envolvidos, aos distintos processos e metodologias pedagógicos e aos projetos e políticas do setor, inseridos em uma conjuntura de mudanças sociais, econômicas e políticas que se operam na atualidade, nos planos nacional e internacional. 
Além disso, as tecnologias da informação e comunicação (TIC) assumiram um papel preponderante no momento atual de pandemia, com destaque para as necessidades de manter as atividades educacionais em ambientes remotos.

A literatura sobre esse campo de pesquisa é muito abrangente e diversificada. Mais recentemente, alguns autores exploraram temas relacionados à política em educação (Martinez \& Machado, 2020), o monitoramento de avaliação dos Planos Decenais de Educação (Aranda, Rodrigues, \& Militão, 2020) e à Base Nacional Comum Curricular (Costa \& Silva, 2019); à formação de professores para a educação infantil (Cunha, Cunha, \& Ferreira, 2020) e para a educação básica (Amaral \& Monteiro, 2019).

Outros autores apresentaram uma análise sobre a regulação da conduta da pesquisa em educação (Savi Neto, Fare, \& Silva, 2020); a caracterização dos passos da Teoria Fundamentada nos Dados (TFD) e as possíveis aplicações na pesquisa em educação (Prigol \& Behrens, 2019); e a relevância para a pesquisa em história da educação (Martins, 2019).

Diante dessa diversidade surge a questão de pesquisa deste estudo: qual o panorama das publicações em português na base Scielo sobre a pesquisa em educação?

\section{Metodologia}

Este trabalho tem como objetivo apresentar um panorama das publicações sobre a pesquisa em educação, por meio de uma análise bibliométrica. Para responder à questão de pesquisa, explorou-se a análise de redes de coocorrência de palavras-chave e de citações de documentos, de periódicos e de organizações. Para isso, foram utilizados métodos de pesquisa bibliométrica (ZUPIC \& CATER, 2014) e de análise de redes (Newman, 2009; Van Eck \& Waltman, 2014).

Uma rede bibliométrica consiste em nós e arestas. Os nós podem ser, por exemplo, publicações, periódicos, organizações, autores ou palavras-chave. As arestas indicam relações entre pares de nós. Os tipos de arestas geralmente mais utilizados são as relações de citação, de co-ocorrência de palavras-chave e de coautoria. No caso de relações de citação, uma distinção adicional pode ser feita entre a citação direta, a cocitação e o acoplamento bibliográfico. As redes bibliométricas são geralmente redes ponderadas. Consequentemente, as arestas indicam não apenas se há uma relação entre dois nós ou não, mas também a força dessa relação.

A análise bibliométrica fornece uma ferramenta útil para inferir conhecimento de um corpo da literatura científica e interpretar a evolução, a distribuição e o desenvolvimento da pesquisa em um determinado campo (Daim, Rueda, \& Martin, 2005). Trata-se de uma abordagem que permite avaliar as relações entre autores, entidades, periódicos ou países, além de medir o impacto da pesquisa e a vinculação envolvendo as co-citações e as palavras-chave empregadas (Lv et al, 2011; Martínez-Gómez, 2015).

Esta pesquisa seguiu as seguintes etapas: desenho da pesquisa com a definição do objetivo e a expressão de busca; escolha das unidades de análise - palavras-chave, documentos, fontes e organizações; escolha do tipo de análise - redes de coocorrência de palavras-chave e de citações; seleção da base Scielo para a pesquisa bibliográfica e a recuperação de informações; escolha do software VOSviewer (Van Eck \& Waltman, 2021) para gerar as redes de coocorrência de palavra-chave e de citações de documentos, fontes e organizações; elaboração do arquivo de tesauros do VOSviewer para controle do vocabulário e normalização de nomes de organizações; obtenção das redes de coocorrência de palavras-chave e de citações de documentos, de fontes e de organizações com o controle do vocabulário e a normalização dos nomes de organizações; escolha do software Gephi (Bastian, Heymann, \& Jacomy, 2009) para o cálculo das métricas de análise de redes - grau médio, classes de modularidade e centralidade de autovetor; identificação dos termos mais relevantes e emergentes dos tópicos de pesquisa; identificação das fontes e das organizações com as maiores centralidades de autovetor. 


\section{Resultados da Pesquisa}

A pesquisa bibliográfica foi realizada na base Scielo Citation Index (Web of Science) utilizando a expressão de busca: educação, que retornou 23731 documentos publicados do período de 2002 a 2021. Como o foco da pesquisa é a análise das publicações em português na área da educação, foram realizados dois refinamentos com a seleção dos seguintes filtros: categoria - education educational research; tipos de documentos - artigos em periódicos e de revisão; e iselecionado o idioma - português.

O resultado é constituído por 7448 documentos publicados no período de 2002 a 2021 . A Figura 1 apresenta a evolução das publicações no período de 2002 a 2021 . Observa-se que o pico de publicações ocorreu em 2018, seguido por 2019 e 2020. A seguir, serão apresentados os resultados das análises de coocorrência e de citações.

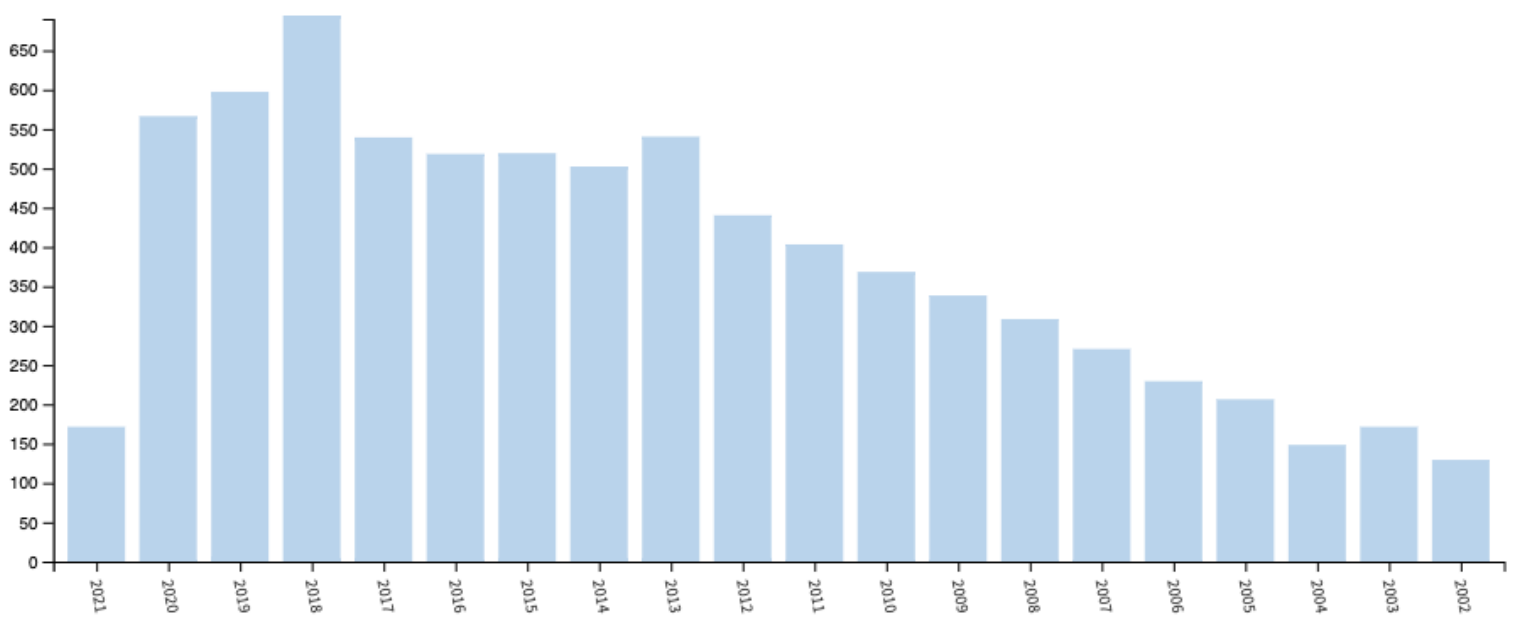

Fig. 1. Evolução das publicações na base Scielo, no período de 2002 a 2021

\subsection{Análise de Coocorrência de Palavras-chave}

Para a obtenção da rede de coocorrência de palavras-chave, foi utilizado o software VOSviewer. As palavras-chave foram extraídas da lista fornecida pelos autores de cada publicação. O número de coocorrências de duas palavras-chave corresponde ao número de publicações nas quais os dois termos ocorreram simultaneamente (Van Eck \& Waltman, 2014).

Os metadados foram lidos e foi selecionada a opção de coocorrência de palavras-chave do autor. Sem o controle do vocabulário, com um mínimo de 1 ocorrência para cada par de palavras-chave, resultou em uma rede de coocorrência com 10694 nós, 11 comunidades e 38783 arestas. Cada comunidade possuía pelo menos 100 nós. Adicionalmente, o VOSviewer oferece uma opção de criar um arquivo de texto para realizar o controle do vocabulário - o tesauro, ou seja, é possível realizar a substituição e a exclusão de termos. Trata-se de uma interpretação qualitativa das palavras-chave incluídas pelos autores nos metadados de cada documento. Com essa opção do controle do vocabulário, a rede resultante incluiu 9011 nós, 11 comunidades e 25473 arestas.

A Figura 2 apresenta a visualização da rede de coocorrência de palavras-chave. É possível identificar algumas palavras-chave que se destacam na rede: special education, physical education, children, teacher training, history of education, medical education, curriculum, distance education, entre outras. Contudo, alguns termos, que aparecem com baixa intensidade e em áreas mais periféricas da figura, podem constituir oportunidades de pesquisa: anti-drug education, educational identity, assessment of higher education, indigenous culture, military education, deaf literature, etc. 


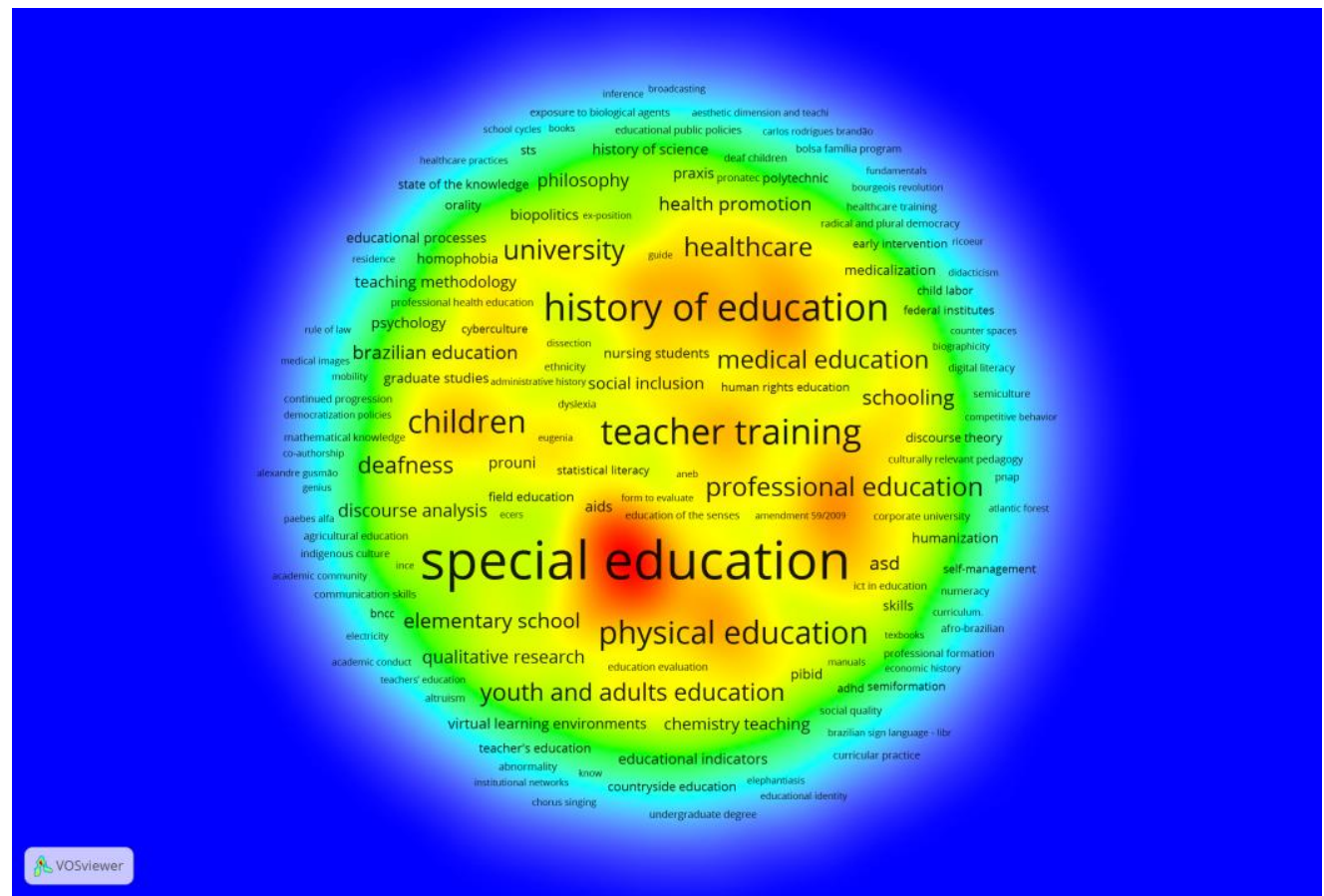

Fig. 2. Rede de coocorrência de palavras-chave dos autores

Contudo, a interpretação desses resultados a partir do mapa de densidade não é precisa. Para obter resultados mais precisos, com a identificação dos termos mais influentes e os emergentes, a rede foi exportada em formato GML para ser recuperada no Gephi (Bastian, Heymann, \& Jacomy, 2009). Em seguida, foram calculadas as métricas de redes: grau médio, modularidade de classes e centralidade de autovetor. A centralidade de autovetor atribui relevância a um nó em função de sua relação com os demais vértices da rede. Se um nó está ligado a outros que se encontram numa posição central na rede, o mesmo terá um valor alto de centralidade de autovetor (Newman, 2009).

A Tabela 1 apresenta os resultados para as 20 palavras-chave com os valores mais elevados de centralidades de autovetor e de grau. A palavra-chave higher edcuation é a que apresenta o maior valor de centralidade de autovetor e o maior grau. Entre as palavras-chave da Tabela 1, algumas merecem destaque: special education, educational policies, curriculum, history of education, basic education, early childhood education, inclusive education, distance education, continued education. Tais palavras-chave são conceitos que mostram a diversidade da pesquisa em educação. Outro ponto a destacar é que estes conceitos têm anos médios de publicação variando de 2012 a 2015.

Para a identificação de temas emergentes, alguns estudos recentes utilizaram a rede de coocorrência da palavras-chave nos seguintes temas: manutenção preditiva e sensores inteligentes em fábricas inteligentes (Pech, Vrchota, \& Bednář, 2021), segurança contra incêndio (Lang et al, 2021), cooperação internacional em migração (Khaldi \& Prado-Gascó, 2021) e saúde e segurança ocupacional e responsabilidade social corporativa (BautistaBernal, Quintana-García, \& Marchante-Lara, 2021).

Neste trabalho, foi utilizado um recurso do VOSviewer que é o ano médio que cada palavra-chave ocorre no conjunto de documentos da pesquisa bibliográfica. Para isso, foi utilizada a rede importada pelo Gephi e extraídas as palavras-chave que tiveram ano médio de 2020, porque não tiveram termos com ano médio de 2021. A Tabela 2 apresenta o resultado para os tópicos emergentes. As palavras-chave foram colocadas em ordem crescente de grau e centralidade de autovetor. Esta ordem revela as palavras-chave com menor grau e baixa influência na rede. A tendência é que, com o tempo e com a publicação de novos documentos, estas palavras-chave se conectem àquelas mais influentes e alcancem relevância por se transformarem em temas de interesse de pesquisa. 
Tabela 1. Palavras-chave mais influentes da rede de coocorrência de palavras-chave.

\begin{tabular}{lccc}
\hline \multicolumn{1}{c}{ Palavra-chave } & Ano Médio & Grau & $\begin{array}{c}\text { Centralidade de } \\
\text { autovetor }\end{array}$ \\
\hline higher education & $2.013,744$ & 721 & 1,0000 \\
special education & $2.013,727$ & 603 & 0,8188 \\
educational policies & $2.012,330$ & 546 & 0,7939 \\
curriculum & $2.014,033$ & 384 & 0,7087 \\
teacher education & $2.012,209$ & 338 & 0,6212 \\
teacher training & $2.014,914$ & 307 & 0,5650 \\
history of education & $2.014,351$ & 390 & 0,5115 \\
basic education & $2.014,280$ & 267 & 0,4887 \\
public policies & $2.012,371$ & 235 & 0,4610 \\
healthcare education & $2.013,028$ & 307 & 0,4555 \\
mathematics education & $2.014,519$ & 337 & 0,4364 \\
early childhood education & $2.015,072$ & 258 & 0,4075 \\
environmental education & $2.012,131$ & 295 & 0,4054 \\
science education & $2.013,187$ & 241 & 0,3967 \\
physical education & $2.015,139$ & 194 & 0,3833 \\
inclusive education & $2.013,738$ & 189 & 0,3507 \\
distance education & $2.012,936$ & 206 & 0,3432 \\
continued education & $2.013,896$ & 150 & 0,3396 \\
high school & $2.013,973$ & 161 & 0,3392 \\
university & $2.012,524$ & 162 & 0,3377 \\
\hline
\end{tabular}

Os resultados revelam a emergência de tópicos atuais, como é o caso da Covid-19, a póspandemia, as inovações pedagógicas e o aprendizado remoto (ou aprendizagem à distância). A realidade atual motivou estudos e pesquisas destes tópicos. O tópico Covid19 se destaca pelo grau e centralidade de autovetor bem maiores que os demais.

\subsection{Análise de Citações}

Vários tipos de relações entre as publicações podem ser usadas para identificar os tópicos de pesquisa, como abordagens baseadas em citações, em textos e híbridas. No caso de abordagens baseadas em texto, alguns estudos estabeleceram clusters com base na coocorrência de termos (Furukawa et al., 2015; Lee, 2008; Rotolo, Hicks, \& Martin, 2015). As abordagens baseadas em citações permitem identificar diversos tipos de relações, tais como: citação direta (Kajikawa \& Takeda, 2008; Kajikawa, Yoshikawa, Takeda, \& Matsushima, 2008; Shibata et al, 2011), acoplamento bibliográfico (Morris, Yen, Wu, \& Asnake, 2003) e cocitação (Small, 2006; Upham \& Small, 2010). Estas relações podem ser usadas para agregar publicações.

Comparadas com a cocitação e o acoplamento bibliográfico, as citações diretas, por vezes chamadas de citações cruzadas, oferecem uma indicação mais imediata da ligação das publicações. No entanto, na literatura sobre visualização de redes bibliométricas, é relativamente incomum trabalhar com citações diretas. Isso provavelmente ocorre porque o uso de citações diretas geralmente leva a redes muito esparsas (ou seja, redes com apenas um número muito pequeno de arestas). Apesar dessa questão, parece haver um interesse crescente em citações diretas na literatura (Boyack \& Klavans, 2010; Persson, 2010; Waltman \& Van Eck, 2012). 
Tabela 2. Palavras-chave emergentes da rede de coocorrência de palavras-chave.

\begin{tabular}{lccc}
\hline \multicolumn{1}{c}{ Palavra-chave } & $\begin{array}{c}\text { Ano } \\
\text { Médio }\end{array}$ & Grau & $\begin{array}{c}\text { Centralidade } \\
\text { de autovetor }\end{array}$ \\
\hline life skills & 2020 & 2 & 0,0007 \\
children's political participation & 2020 & 2 & 0,0010 \\
education of refugees & 2020 & 2 & 0,0010 \\
evidence-based education & 2020 & 2 & 0,0013 \\
ethnic-racial relationships & 2020 & 2 & 0,0019 \\
exclusive school & 2020 & 2 & 0,0022 \\
remote learning & 2020 & 2 & 0,0034 \\
post-pandemic & 2020 & 2 & 0,0111 \\
education of deaf children & 2020 & 2 & 0,0121 \\
military school & 2020 & 2 & 0,0153 \\
student with high abilities & 2020 & 2 & 0,0188 \\
anti-drug education & 2020 & 3 & 0,0043 \\
drugs at school & 2020 & 3 & 0,0043 \\
ethical violence & 2020 & 3 & 0,0052 \\
fake news & 2020 & 3 & 0,0092 \\
digital technologies of information and communication & 2020 & 3 & 0,0186 \\
pedagogical innovations & 2020 & 4 & 0,0021 \\
computational thinking & 2020 & 4 & 0,0112 \\
digital games & 2020 & 4 & 0,0112 \\
covid-19 & 2020 & 16 & 0,0423 \\
\hline
\end{tabular}

Foram geradas as redes de citação de documentos, de fontes e de organizações, ambas no VOSviewer e exportadas para o Gephi. A rede de citação de documentos obtida, com o mínimo de 5 citações por documento, possui 376 nós, 19 comunidades e 459 arestas. A Tabela 3 apresenta os 10 artigos mais influentes da rede e os seus respectivos tópicos de pesquisa. Nota-se que, apesar dos artigos possuirem grau baixo, têm centralidades de autovetor mais alta.

Tabela 3. Os 10 artigos mais influentes da rede de citação de citação de documentos.

\begin{tabular}{|c|c|c|c|}
\hline Documento & Grau & $\begin{array}{l}\text { Centralidade } \\
\text { de autovetor }\end{array}$ & Tópico de pesquisa \\
\hline Dourado (2013) & 2 & 1,000 & sistema nacional de educação \\
\hline Dourado (2015) & 9 & 0,913 & diretrizes curriculares nacionais \\
\hline Frigotto, Ciavatta, \& Rmos (2005) & 2 & 0,857 & educação profissional \\
\hline Jacomini \& Penna (2016) & 2 & 0,710 & $\begin{array}{c}\text { carreira docente - educação } \\
\text { básica }\end{array}$ \\
\hline Gatti (2013) & 1 & 0,700 & formação de professores \\
\hline Afonso (2013) & 6 & 0,666 & avaliação da educação \\
\hline Barreyro \& Rothen (2014) & 7 & 0,568 & avaliação da educação superior \\
\hline Oliveira (2004) & 9 & 0,541 & $\begin{array}{c}\text { carreira docente - educação } \\
\text { básica }\end{array}$ \\
\hline Ristoff (2014) & 2 & 0,535 & $\begin{array}{c}\text { democratização do ensino } \\
\text { superior }\end{array}$ \\
\hline
\end{tabular}


A rede de citação de fontes foi gerada pelo VOSviewer e importada pelo Gephi. Neste caso, as a opção foi a de indagar quais os periódicos que publicaram os artigos. A rede de citação de fontes obtida, com o mínimo de 1 citação por periódico, possui 42 nós, 4 comunidades e 271 arestas. A Tabela 4 apresenta os 10 periódicos mais influentes desta rede. Observa-se que o periódico mais influente possui classificação B2 no Qualis da Capes. Contudo, sete periódicos são A1, e os outros dois, A2 e A4.

Tabela 4. Os 10 periódicos mais influentes na rede de citação de fontes.

\begin{tabular}{lccc}
\hline \multicolumn{1}{c}{ Periódico } & Grau & $\begin{array}{c}\text { Centralidade } \\
\text { de autovetor }\end{array}$ & $\begin{array}{c}\text { Qualis/ } \\
\text { Capes }\end{array}$ \\
\hline Trabalho, Educação e Saúde & 20 & 1,0000 & B2 \\
Revista Portuguesa de Educação & 14 & 0,8809 & A1 \\
Revista Brasileira de Estudos Pedagógicos & 18 & 0,7569 & A1 \\
Revista Brasileira de Educação Especial & 21 & 0,7156 & A1 \\
Revista Brasileira de Educação & 32 & 0,6766 & A1 \\
Revista Lusófona de Educação & 12 & 0,5724 & A1 \\
Pro-posições & 24 & 0,4701 & A1 \\
Revista Electrónica de Investigación en Educación en & & & A2 \\
Ciencias & 8 & 0,3373 & A1 \\
Sociologia \& Antropologia & 3 & 0,2973 & A4 \\
Interface - Comunicação, Saúde, Educação & 18 & 0,2617 & \\
\hline
\end{tabular}

A última rede gerada foi a de citação de organizações, que foi opbtida pelo VOSviewer e exportada para o Gephi. A Figura 3 apresenta o mapa de densidade de citações das organizações. Em destaque aparecem as seguintes instituições: Universidade de São Paulo (USP), Universidade Estadual de São Paulo (UNESP), Universidade Estadual de Campinas (Unicamp), Universidade Federal do Rio Grande do Sul (UFRGS), Universidade Federal do Rio de Janeiro (UFRJ), Universidade Federal do Paraná (UFPR), Universidade Federal de São Carlos (UFSCar), Universidade Federal de Minas Gerais (UFMG), Universidade Federal de Santa Maria (UFSM), entre outras.

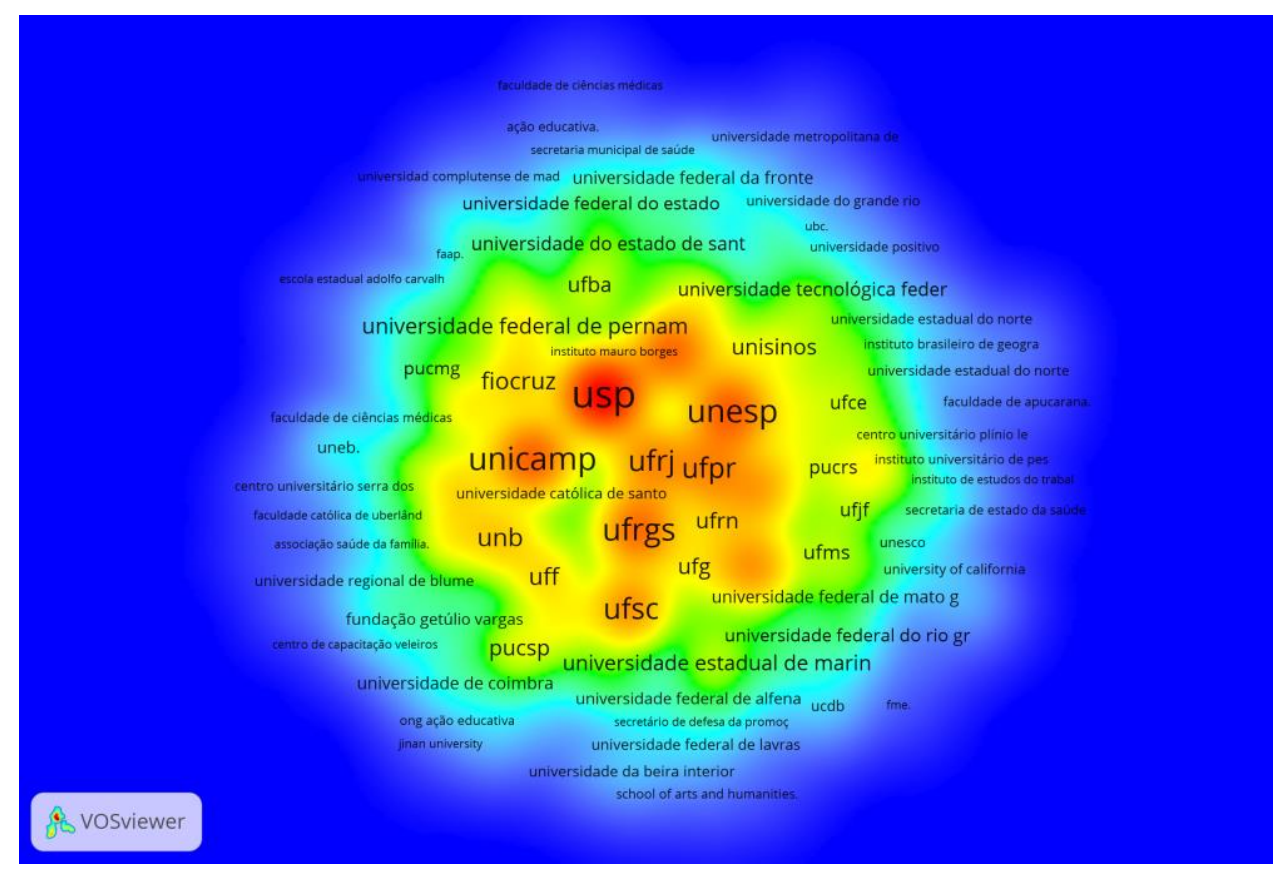

Fig. 3. Rede de citação de organizações 
Para verificar as organizações ou instituições mais influentes na rede, a Tabela 5 apresenta a rede de citação de organizações. Observa-se que algumas instituições da Figura 3 constam da Tabela 5, tais como USP, UFSCar e UFSM. Como a influência é determinada pela centralidade de autovetor, insituições que não ficaram visíveis na Figura 3 constam desta Tabela. Outro ponto a destacar é que oito instituições são brasileiras, uma da Espanha e uma do Reino Unido. Como o recorte foi feito no idioma português, o resultado está coerente.

Tabela 5. As 10 organizações mais influentes na rede de citação de fontes.

\begin{tabular}{lccc}
\multicolumn{1}{c}{ Organização } & Grau & $\begin{array}{c}\text { Centralidade } \\
\text { de autovetor }\end{array}$ & País \\
\hline Universidade de São Paulo & 210 & 1,0000 & Brasil \\
Universidade Metodista de Piracicaba & 66 & 0,4363 & Brasil \\
Universidade Federal de São Carlos & 129 & 0,4339 & Brasil \\
Universidade Federal do Espírito Santo & 87 & 0,3849 & Brasil \\
Universitat de Barcelona & 45 & 0,3463 & Espanha \\
Universidade Federal Do Pará & 66 & 0,3328 & Brasil \\
Universidade Tecnológica Federal do Paraná & 52 & 0,3148 & Brasil \\
Universidade Federal de Pernambuco & 99 & 0,3036 & Brasil \\
University of London & 34 & 0,2838 & Reino Unido \\
Universidade Federal de Santa Maria & 87 & 0,2836 & Brasil \\
\hline
\end{tabular}

\section{Conclusões}

Esta pesquisa apresentou um panorama da pesquisa em educação a partir da análise da bibliografia recuperada na base Scielo Citation Index (via Web of Science).De notar que a produção em língua portuguesa é mais vasta do que indexada nesta base referencial. Futuras pesquisas pretendem ultrapassar esta limitação para dar visibilidade a essa produção, aos seus autores, instituições e países que divulgam o conhecimento em língua portuguesa.

Como o campo de pesquisa é muito vasto, a metodologia utilizada mostra um caminho para que seja obtido um panorama usando métodos da análise de redes bibliométricas.

A partir desta base de trabalho, onde foram identificados tópicos de pesquisa mais relevante e emergentes, além dos periódicos e das organizações mais influentes, surgem novas questões:

- quais os tópicos pesquisados pelas organizações mais influentes?

- quais os autores mais relevantes e suas contribuições para a pesquisa em educação?

- qual a cooperação e os tópicos de pesquisa existentes entre as organizações mais influentes?

O desafio de dar respostas a essas questões indica novos aprofundamentos empregando métodos qualitativos para a interpretação dos resultados. Este grupo de artigos pode ser complementado por outros recuperados nas bases Scopus e Web of Science, a partir dos refinamentos utilizados neste trabalho. A interpretação dos artigos relevantes pode ser objeto de análise de conteúdo, agilizada pelo Software WebQDA, alinhada com as questões que agora emergiram (Costa \& Amado, 2018). 
A potencialidade de questionamento deste software irá permitir um rico aprofundamento na exploração dos textos dos artigos relevantes.

Deste modo, apesar das inerentes limitações deste estudo exploratório, ele se revela de grande utilidade para desenhar e realizar uma revisão da literatura, sobre um subtema que queiramos focar. O mapeamento agora obtido assume uma importância por apresentar indicações de rotas de pesquisa num campo tão vasto e diversificado como é a Pesquisa em Educação.

\section{Referências}

Afonso, A. J. (2013). Mudanças no Estado-avaliador: comparativismo internacional e teoria da modernização revisitada. Revista Brasileira de Educação, 18(53), 267-284

Amaral, M. H., \& Monteiro, M. I. B. (2019). Teacher Education on GT 15 - Anped's Special Education (2011-2017) between Dialogues and (New) Clues. Revista Brasileira de Educação Especial, 25(2), 301-318.

Aranda, M. A. M., Rodrigues, E. S. S., \& Militão, S. C. N. (2020). Monitoring and Evaluation of Decennial Education Plans: the production of knowledge in Brazil. Educar em Revista, 36, e69767.

Barreyro, G. B., \& Rothen, J. C. (2014). Percurso da avaliação da educação superior nos Governos Lula. Educação e Pesquisa, 40(1), 61-76.

Bastian, M., Heymann, S., \& Jacomy, M. (2009). Gephi: An Open Source Software for Exploring and Manipulating Networks. In: Proceedings of the Third International ICWSM Conference, 361-362.

Bautista-Bernal, I., Quintana-García, C., \& Marchante-Lara, M. (2021). Research trends in occupational health and social responsibility: A bibliometric analysis. Safety Science, 137, Art. 105167

Boyack, K.W., \& Klavans, R. (2010). Co-citation analysis, bibliographic coupling, and direct citation: Which citation approach represents the research front most accurately? Journal of the American Society for Information Science and Technology, 61(12), 2389-2404.

Costa, A. P., \& Amado, J. (2018). Análise de Conteúdo Suportada por Software: Ludomedia.

Costa, M. O., \& Silva, L. A. (2019). Educação e democracia: Base Nacional Comum Curricular e novo ensino médio sob a ótica de entidades acadêmicas da área educacional. Revista Brasileira de Educação, 24, e240047.

Cunha, N. V. S., Cunha, M. L., \& Ferreira, H. S. (2020). Concepção de formação humana para a educação infantil: um estado da questão. Revista Brasileira de Educação, 25, e250033.

Daim, T. U., Rueda, G. R., \& Martin, H. T. (2005). Technology forecasting using bibliometric analysis and system dynamics. In Technology management: In a unifying discipline for melting the boundaries - Conference Proceedings - IEEE, 112-122.

Dourado, L. . (2013). Sistema Nacional de Educação, Federalismo e os obstáculos ao direito à educação básica. Educação \& Sociedade, 34(124), 761-785.

Dourado, L. F. (2015). Diretrizes curriculares nacionais para a formação inicial e continuada dos profissionais do magistério da educação básica: concepções e desafios. Educação \& Sociedade, 36(131), 299-324.

Frigotto, G., Ciavatta, M., \& Ramos, M. (2005). A política de educação profissional no Governo Lula: um percurso histórico controvertidoProfessional education policy in the Lula's administration: a controverted historical trajectory. Educação \& Sociedade, 26(92), 10871113.

Furukawa, T., Mori, K., Arino, K., Hayashi, K., \& Shirakawa, N. (2015). Identifying the evolutionary process of emerging technologies: A chronological network analysis of World Wide Web conference sessions. Technological Forecasting \& Social Change, 91(2), 280- 294.

Gatti, B. A. (2013). Educação, escola e formação de professores: políticas e impasses. Educar em Revista, (50), 51-67. 
Jacomini, M. A., \& Penna, M. G. O. (2016). Carreira docente e valorização do magistério: condições de trabalho e desenvolvimento profissional. Pro-Posições, 27(2), 177-202.

Kajikawa, Y., \& Takeda, Y. (2008). Structure of research on biomass and bio-fuels: A citationbased approach. Technological Forecasting \& Social Change, 75(9), 1349-1359.

Kajikawa, Y., Yoshikawa, J., Takeda, Y., \& Matsushima, K. (2008). Tracking emerging technologies in energy research: Toward a roadmap for sustainable energy. Technological Forecasting \& Social Change, 75(6), 771-782.

Khaldi, H., \& Prado-Gascó, V. (2021). Bibliometric maps and co-word analysis of the literature on international cooperation on migration. Quality \& Quantity, Article in Press.

Lang, Z., Liu, H., Meng, N., Wang, H., Wang, H., \& Kong, F. (2021). Mapping the knowledge domains of research on fire safety - an informetrics analysis. Tunnelling and Underground Space Technology, 108, Art. 103676.

Lee, W. H. (2008). How to identify emerging research fields using scientometrics: An example in the field of Information Security. Scientometrics, 76(3), 503-525.

Lv, P. H., Wang, G.-F., Wan, Y., Liu, J., Liu, Q., \& Ma, F. (2011). Bibliometric trend analysis on global graphene research. Scientometrics, 88(2), 399-419.

Martinez, C. T., \& Machado, A. M. (2021). Diálogos entre o método da cartografia e alguns pressupostos filosóficos de Hannah Arendt na pesquisa em educação. Interface Comunicação, Saúde, Educação, 25, e190835.

Martínez-Goómez, A. (2015). Bibliometrics as a tool to map uncharted territory: A study on nonprofessional interpreting. Perspectives, 23(2), 205-222.

Martins, E. C. R. (2019). History, historiography and research in historical education. Educar em Revista, 35(74), 17-33.

Morris, A. S., Yen, G., Wu, Z., \& Asnake, B. (2003). Timeline visualization of research fronts. Journal American Society for Information Science and Technology, 54(5), 413-422.

Newman, M. (2009). Networks: an introduction. Oxford: Oxford University Press.

Oliveira, D. A. (2004). A reestruturação do trabalho docente: precarização e flexibilização. Educação \& Sociedade, 25(89), 1127-1144.

Pech, M., Vrchota, J., \& Bednář, J. (2021). Predictive Maintenance and Intelligent Sensors in Smart Factory: Review. Sensors, 21(4), Art. 1470, 1-40.

Persson, O. (2010). Identifying research themes with weighted direct citation links. Journal of Informetrics, 4(3), 415-422.

Prigol, E. L., \& Behrens, M. A. (2019). Grounded Theory: methodology applied in education research. Educação \& Realidade, 44(3), e84611.

Ristoff, D. (2014). O novo perfil do campus brasileiro: uma análise do perfil socioeconômico do estudante de graduação. Avaliação: Revista da Avaliação da Educação Superior, 19(3), 723747.

Rotolo, D., Hicks, D., \& Martin, B. R. (2015). What is an emerging technology? Research Policy, 44(10), 1827-1843.

Savi Neto, P., Fare, M., \& Silva, D. S. (2020). Ethics, autonomy and research in education: raising questions about Brazilian regulations on the conduct of researchers. Revista Brasileira de Educação, 25, e250013.

Shibata, N., Kajikawa, Y., Takeda, Y., Sakata, I., \& Matsushima, K. (2011). Detecting emerging research fronts in regenerative medicine by the citation network analysis of scientific publications. Technological Forecasting \& Social Change, 78(2), 274-282.

Small, H. (2006). Tracking and predicting growth areas in science. Scientometrics, 68(3), 595- 610.

Upham, S. P., \& Small, H. (2010). Emerging research fronts in science and technology: Patterns of new knowledge development. Scientometrics, 83(1), 15-38. 
Van Eck, N. J., \& Waltman, L. (2014). Visualizing bibliometric networks. In: Ding, Y., Rousseau, R., \& Wolfram, D. (Eds.). Measuring scholarly impact: methods and practice. New York: Springer.

Van Eck, N. J., \& Waltman, L. (2021). VOSviewer manual. Leiden: Universiteit Leiden.

Waltman, L., \& Van Eck, N.J. (2012). A new methodology for constructing a publication-level classification system of science. Journal of the American Society for Information Science and Technology, 63(12), 2378-2392.

Zupic, I., \& Cater, T. (2014). Bibliometric methods in management organization. Organizational Research Methods, 18 (3), 429-472. 